\title{
A LATERAL INTERMUSGULAR APPROACH TO THE HIP JOINT FOR REPLACEMENT OF THE FEMORAL HEAD BY A PROSTHESIS
}

\author{
H. N. Burwell and Dan Scott, Leeds, England
}

From the Orthopaedic Department of the General Infirmary at Leeds

The purpose of this communication is to present a simple method of exposure of the hip joint for the insertion of an artificial femoral head in patients who have fracture of the femoral neck or osteoarthritis of the hip, many of whom are old or feeble.

For fractures of the femoral neck, the prevalent view seems to be that operation should always be undertaken unless the patient is moribund or suffering from advanced senile mental change (Williams and McLean 1952). Hitherto it has been thought that a rapid extra-articular nailing operation disturbs the patient least. Replacement of the femoral head by a prosthesis, through the exposure described here, is considered to be no more dangerous than an extra-articular nailing operation.

In the treatment of osteoarthritis of the hip, many patients are over seventy years of age and the natural tendency is to avoid the risk of surgery. Replacement of the femoral head by way of this lateral intermuscular approach proves not to be a severe ordeal.

Essentially, the surgical approach advocated here is a modification of that described by Watson-Jones (1936), in which neither muscle nor tendon is divided.

\section{THE OPERATION}

The patient is placed in a lateral position with the affected hip uppermost. Rests are used to stabilise the patient by placing one before the abdomen, one behind the lower lumbar region and one behind the thorax. The surgeon stands behind the patient.

The skin incision is angled; its lower limit lies over the anterior border of the upper six inches of the femoral shaft and the upper limit passes upwards and backwards towards a point on the iliac crest three inches in front of the posterior superior iliac spine (Fig. 1). It is unnecessary to undercut the skin. The ilio-tibial tract and gluteal aponeurosis are incised in the same line as the skin incision, along the antero-lateral aspect of the shaft, and upwards and backwards along the upper border of the gluteus maximus (Fig. 2). The posterior flap of fascia lata is reflected backwards to expose the insertions of the glutei on the trochanter (Fig. 3). With an assistant holding the foot so that the thigh is in lateral rotation the anterior flap is reflected forwards on a deep plane to expose, successively, the anterior surface of the vastus lateralis, the capsule of the joint, the surface of the gluteus medius, and the division between the anterior borders of the glutei and the tensor fasciae latae almost up to the iliac crest (Fig. 4). The dissection is made close to the capsule, the pad of fat containing branches of the lateral circumflex vessels being held forwards by a retractor. The nerve to tensor fasciae latae runs forwards from the glutei deeply, close to the ilium, about one inch from the crest.

The exposure of the anterior capsule is then completed medially, again close to the capsular fibres, until the rim of the acetabulum is reached. This limit is determined by one, or a combination, of three ways. If the fibres of iliacus are exposed in the dissection, the lateral border is retracted anteriorly and medially. If the limb is gently rocked, the margin of the acetabulum can be palpated as a fixed structure in contrast to the moving head. If the capsule is incised along the middle of the neck the labrum is reached (Fig. 4). 


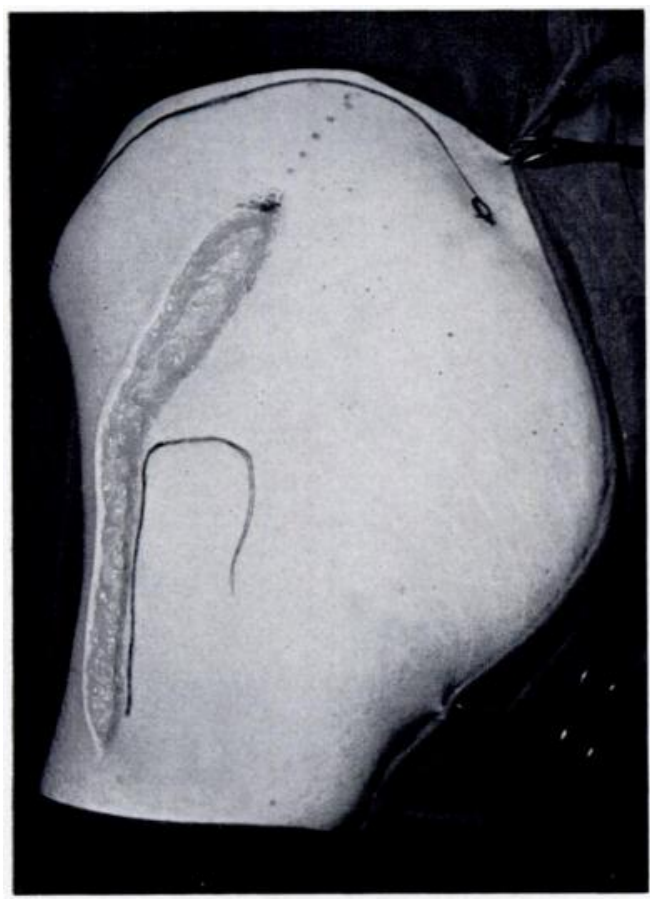

Fig. 1

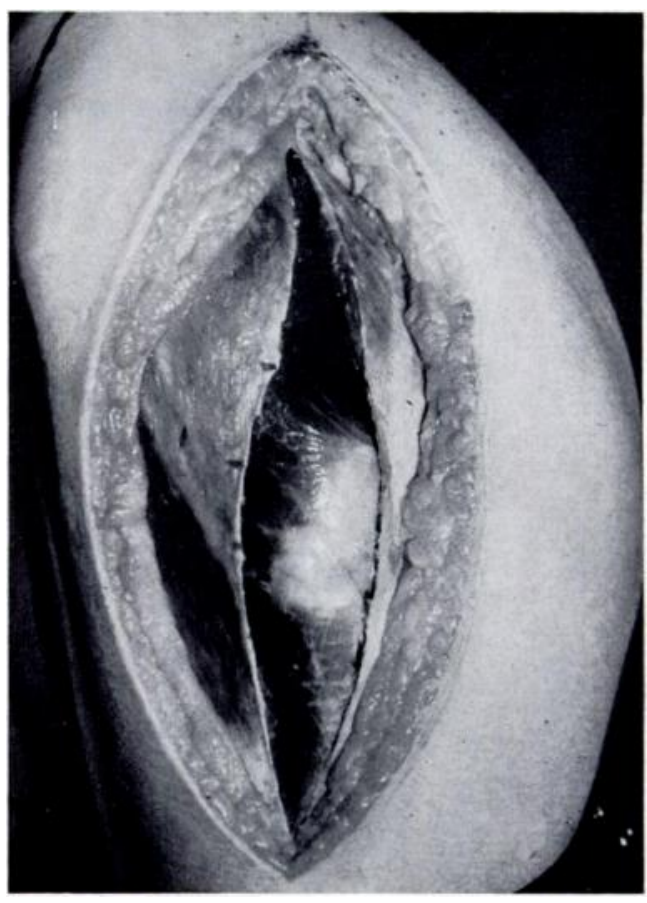

Fig. 2

Figure 1-The skin incision. Its upper limit is three inches in front of the posterior superior spine of the ilium. Figure 2-The fascia lata has been incised: the gluteus maximus has fallen backwards and the tensor fasciae latae forwards, exposing the gluteus medius, the greater trochanter and vastus lateralis.

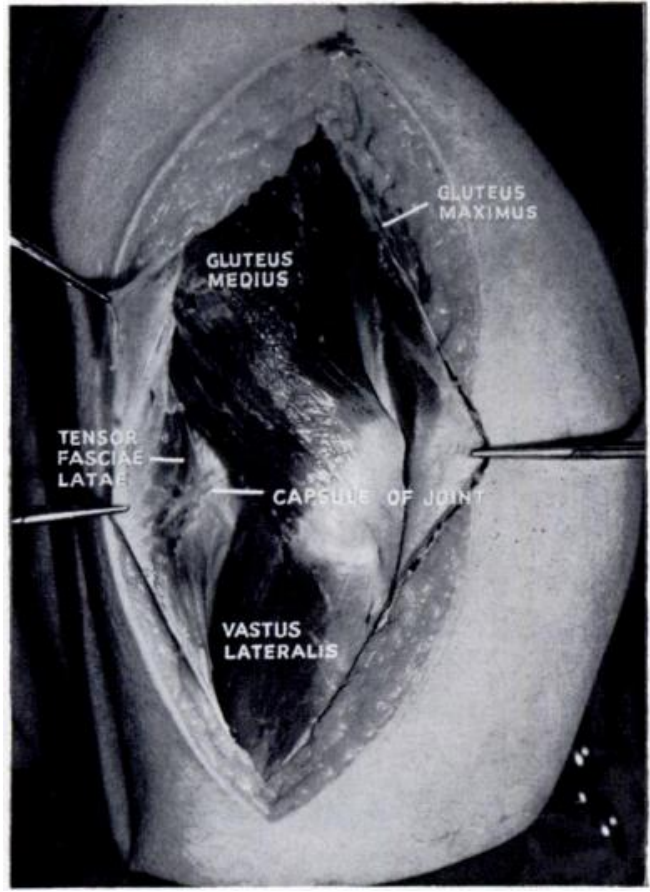

Fig. 3

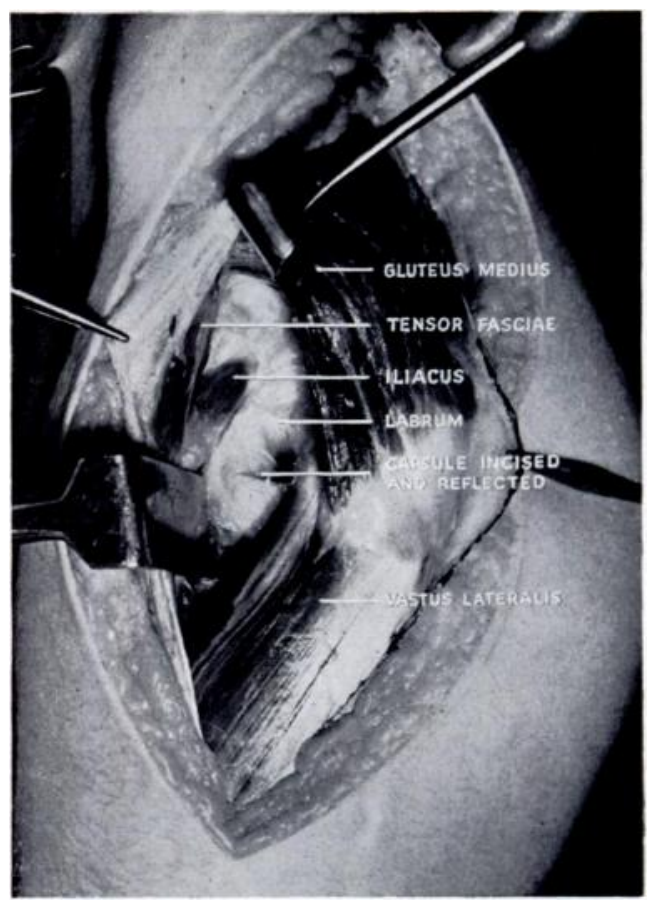

FIG. 4

Figure 3-Further reflection of gluteus maximus backwards and tensor fasciae latae forwards uncovers the whole width of gluteus medius and reveals the capsule of the hip joint. Figure 4-The capsule has been incised and reflected, to reveal the labrum.

vol. 36 B, No. 1, FEBRUARY 1954 
The capsule is either excised or opened by an H-shaped incision and retracted (Fig. 5). The strong superior part of the capsule is divided as it runs downwards and laterally deep to the anterior border of gluteus minimus.

The hip is dislocated by the following manoeuvre. The knee is flexed with the hip maintained in extension. The femur is abducted, and then rotated laterally so that the foot is brought to the opposite knee. In this way lateral rotation is obtained without unduly stretching the glutei. Rotation is maintained while the foot of one limb is moved down the shin of the other, so that abduction is reduced. The last movements must be performed carefully so that the head, or fractured neck, emerges in front of the glutei without damaging them (Fig. 6).

If the neck of the femur is broken, the femoral head is removed from the acetabulum.

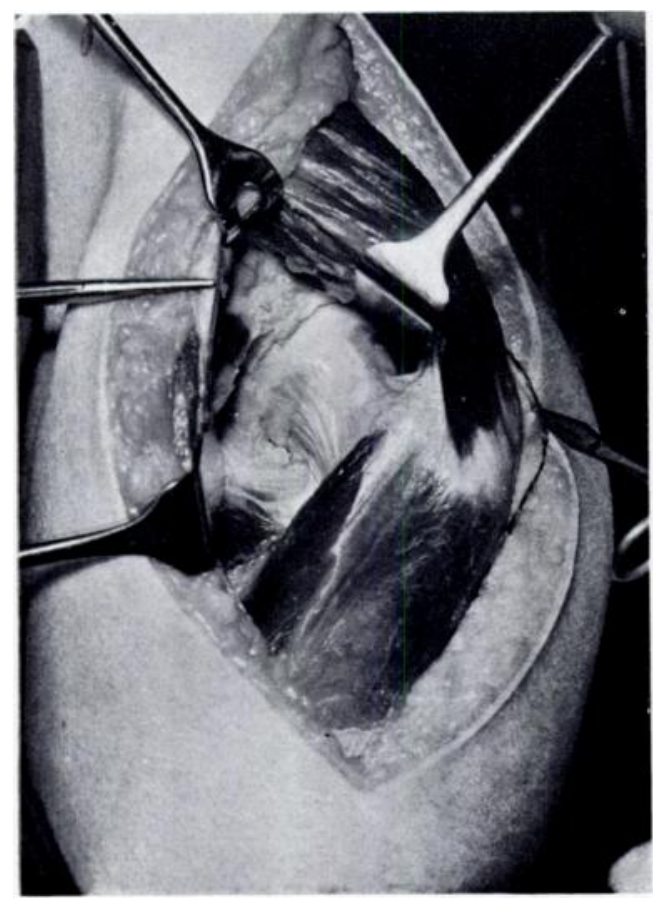

FIG. 5

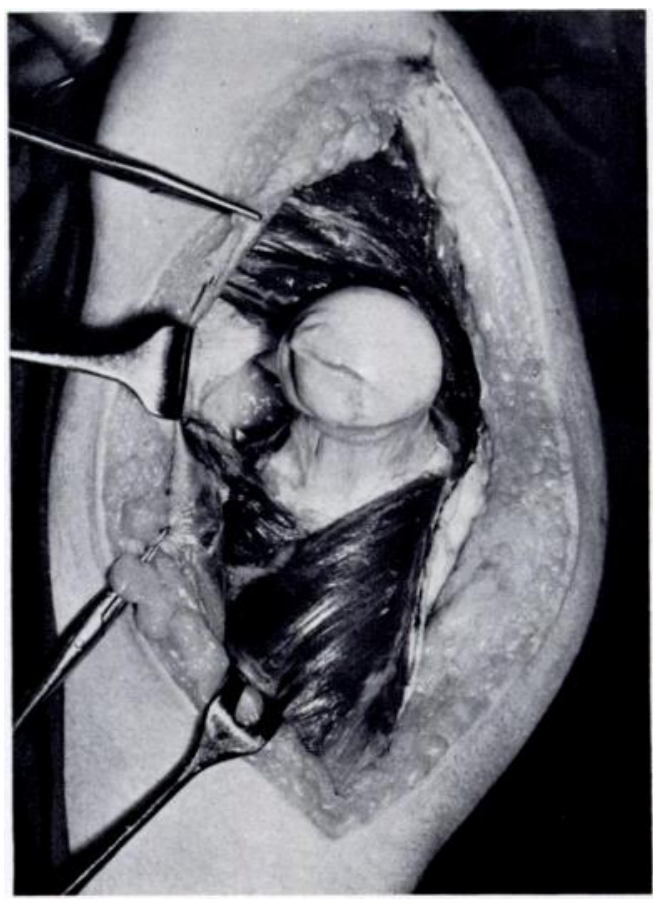

FIG. 6

Figure 5-The labrum has been excised in preparation for dislocating the joint. Figure 6-The joint dislocated and the femoral head turned forwards.

The prosthesis is fitted according to one of the usual techniques. The hip is reduced by traction and medial rotation with the hip and knee extended.

The wound is closed in two layers with interrupted sutures for the fascia lata and gluteal aponeurosis. Usually it is unnecessary to employ any splint.

Special points in the operation-It is important to secure the patient in the correct position on the table before beginning the operation. This facilitates accurate placing of the incision and dislocation of the hip.

Exposure of the neck of the femur between tensor fasciae latae and the glutei has been described for open reduction of fractures (Watson-Jones 1936, Hudson and Bartels 1939). In that operation it is not necessary to dislocate the hip and the incision through the skin and fascia is accord ngly made in the plane of separation of the muscles, between the greater trochanter and the anterior superior iliac spine. With such an incision, the posterior flap of fascia lata tightens on lateral rotation of the hip and prevents easy dislocation of the joint. 
The incision described by Gibson (1950) permits easier rotation of the femur, and after dislocation the head presents in the centre of the wound. The method of dislocating the hip described by Gibson involves flexion of the joint, and it has been found that this movement causes excessive tension on the intact glutei. In dislocating the hip by the intermuscular method, flexion is therefore avoided and it is at this stage that the help of the anaesthetist may be sought to obtain temporary muscle relaxation. Despite these precautions a few of the muscle fibres of the glutei may be torn. This is found not to impair the final result.

\section{DISCUSSION}

The intermuscular approach described is basically that of Watson-Jones (1936), the difference being that the upper part of the incision is inclined backwards instead of forwards and that the head of the femur is dislocated. This approach was adopted for the replacement of the femoral head after a trial of that described by Gibson (1950), in which the insertions of the glutei are severed near their attachments to the greater trochanter and turned up to expose the hip. Gibson's approach gives an excellent exposure but at the conclusion of the operation the glutei, which form an important group of hip stabilisers, have to be repaired. The tendon repair involves the disadvantage of delay to await healing. There is also the risk of permanent muscle weakness and possibly failure of union: it has been found that the wide postero-lateral exposure tends to cause a Trendelenberg type of limp (Nissen 1952). For this reason some surgeons prefer the short vertical approach of Hueter, advocated by Judet and Judet (1950), which, like the lateral intermuscular approach, has the advantage of dividing neither muscle nor tendon. We find that the lateral intermuscular approach gives a better exposure than the short anterior one of Hueter.

In the use of the artificial femoral head for osteoarthritis of the hip many surgeons consider that it is usually unnecessary to remove osteophytes from the rim of the acetabulum. If unexpected difficulty is encountered in delivering the head of the femur because of osteophytic formation, or if it is found at operation that osteophytes are likely to limit movement, then those osteophytes that are accessible from the front may be removed. If exceptional difficulty were to be found, it would be a simple matter to extend the lateral intermuscular approach by division of the gluteal tendons (Gibson 1950) or by separation of their bony insertion (Postel 1951).

Operation by the lateral intermuscular approach causes little or no shock. Blood loss is negligible: no large vessel is visible, and usually haemostats need to be applied to subcutaneous vessels only. The time taken for the operation is short, a matter of great importance to old people. The supposition that the manoeuvre of dislocating the hip is attended by considerable shock has been disproved by pulse and blood-pressure records during operation.

The after-care of a patient with a fracture of the femoral neck which has been operated on through the lateral intermuscular approach is simple. Spontaneous dislocation of the hip is unlikely because forced lateral rotation would be required to produce it. The patient may be allowed to sit out of bed the day after operation, and hip movements are started about ten days later, when the wound is healed. At that time, or within a few days, the patient begins to walk with crutches. A good range of hip movement is usually regained rapidly. After operation for osteoarthritis, remedial treatment must be more intensive to restore mobility and to redevelop the abductors and extensors of the hip, because the muscles are weak from disuse. But walking with crutches need not be delayed on this account.

We believe that replacement of the femoral head, through the lateral intermuscular approach, is a sound primary operation for fracture of the femoral neck in elderly or unfit patients. The operation is no more severe than that of nailing. So far we have used the lateral intermuscular approach in more than thirty operations for osteoarthritis or fracture of the femoral neck, and in four operations for open reduction of congenitally dislocated hips.

Vol. $36 \mathrm{~B}$, No. 1, FEBRUARY 1954 


\section{SUMMARY}

1. A lateral intermuscular approach to the hip joint is described for replacement of the head of the femur by a prosthesis.

2. Advantages claimed for it are that it is attended by little or no shock, because the operation is rapid and relatively bloodless; that adequate exposure can be gained without dividing muscle or tendon; and that muscle function is unimpaired and so walking can be allowed early. The exposure is easily extended if unexpected difficulty is encountered.

3. Prosthetic replacement of the femoral head through this incision is advocated as a primary measure for elderly or unfit patients with fractured femoral neck.

We wish to thank Mr R. Broomhead, Mr A. B. Pain and Mr J. M. P. Clark for their encouragement and kindly criticism, and for permission to operate on some of the patients. Our thanks are due to Mr A. Pegg of the Department of Photography of the University of Leeds.

\section{REFERENCES}

Gibson, A. (1950): Posterior Exposure of the Hip Joint. Journal of Bone and Joint Surgery, 32-B, 183. Hudson, O. C., and Bartels, W. P. (1939): Open Fixation of Intracapsular Fracture of the Femur: An Improved Technique. Journal of Bone and Jount Surgery, 21, 1,001.

Judet, J., and Judet, R. (1950): The Use of an Artificial Femoral Head for Arthroplasty of the Hip Joint. Journal of Bone and Joint Surgery, 32-B, 166.

Nissen, K. I. (1952): The Judet Arthroplasty of the Hip via Gibson's Lateral Approach. Postgraduate Medical Journal, 28, 412.

Postel, M. (1951): Arthroplasties de la Hanche. Paris: Encyclopédie Médico-Chirurgicale.

Watson-Jones, R. (1936) : Fractures of the Neck of the Femur. British Journal of Surgery, 23, 787.

Williams, D. O., and McLean, E. K. (1952): The Treatment of Recent Transcervical Fractures of Femur. British Medical Journal, i, 580. 